\title{
LA INNOVACIÓN Y EL DESARROLLO TECNOLÓGICO EN LA POLÍTICA REGIONAL DE LA UNIÓN EUROPEA, 2000-2006. UN ANÁLISIS DE LAS REGIONES MENOS DESARROLLADAS
}

\author{
Margarita Argüelles Vélez \\ Profesora de Universidad en el Departamento de Economía Aplicada de la Universidad de Oviedo. \\ Doctora em Economía por La Universidad de Oviedo. \\ marguel@uniovi.es
}

\section{Carmen Benavides González}

Profesora de Universidad en el Departamento de Economía de la Universidad de Oviedo.

Doctora em Economía por La Universidad de Oviedo.

cbenavi@uniovi.es

\section{Matías Mayor Fernández}

Profesor de Universidad en el Departamento de Economía Aplicada de la Universidad de Oviedo. Doctor en economía em Economía por La Universidad de Oviedo.

mmayorf@uniovi.es

\section{RESUMEN}

El triángulo del conocimiento formado por investigación-educación-innovación está en la base de los esfuerzos europeos para lograr los ambiciosos objetivos de la Estrategia de Lisboa: convertir a la economía de la Unión Europea (UE) en la economía basada en el conocimiento más competitiva y dinámica del mundo. La UE aplica diferentes políticas para alcanzar estos objetivos, entre las cuales se encuentra la política de cohesión económica y social. Esta se dirige a reducir las disparidades entre las regiones europeas, especialmente promoviendo el crecimiento de las regiones menos desarrolladas y de todas aquellas que experimentan serios cambios estructurales. El ámbito del estudio que aquí se presenta son precisamente las regiones menos desarrolladas de la UE-15 (antes de la ampliación hacia el Este de Europa), prestando una especial atención a las de España y Portugal. Para estas regiones se analizan los programas operativos de los Fondos Estructurales correspondientes al último periodo de programación finalizado: 2000-2006. El objetivo del trabajo es evaluar el impacto del esfuerzo realizado en la creación y mejora de capacidades regionales de investigación e innovación, incluyendo las nuevas TICs, sobre el crecimiento económico regional. Pero el desarrollo de la sociedad del conocimiento no es la única partida de gasto contemplada en los programas operativos de desarrollo regional analizados, por lo que se estudiará el peso de los distintos ejes de actuación, entre los que se incluyen, la mejora de la competitividad, especialmente de las PYMEs, la protección del medio ambiente, la inversión en infraestructuras de transporte y energía y el desarrollo local y urbano o rural.

Palabras-clave: Desarrollo tecnológico; Innovación; Política de innovación; Desarrollo regional. 


\section{INTRODUCCIÓN}

La Unión Europea (UE), en un contexto de ampliación de sus fronteras y de avances en su proceso de integración (consolidación del mercado único e introducción del euro), ha ido desarrollando políticas orientadas a mejorar su competitividad en un entorno mundial cada vez más globalizado, pero sin olvidar también la cohesión económica y social. De hecho, la política regional, dirigida a reforzar la cohesión interna, persigue mejorar la competitividad de todo el territorio comunitario, haciendo un especial hincapié en aquellas regiones más desfavorecidas en términos relativos. Para alcanzar este objetivo, la política de cohesión invierte en distintos ámbitos, tales como la mejora de la competitividad empresarial, la sociedad de la información, la mejora de los recursos humanos o la dotación de infraestructuras, entre otros.

En este trabajo se pretende estimar el impacto de los programas de los Fondos estructurales ejecutados en un conjunto de regiones europeas objetivo $\mathrm{n}^{\circ} 1$-esto es, las regiones menos desarrolladas de la Comunidad- en el periodo 2000-2006. Se trata de determinar si estas actuaciones han tenido un impacto positivo sobre el crecimiento económico regional y, en tal caso, qué tipo de acciones muestran una mayor influencia. Atención especial se prestará a las medidas destinadas a favorecer la sociedad del conocimiento, principalmente en España y Portugal.

El trabajo se estructura de la siguiente forma: en la segunda sección, se introduce la política de cohesión económica y social en la UE; en la tercera, se presentan los datos que han servido de base para el estudio realizado; en la cuarta, se estiman dos modelos con el fin de cuantificar el impacto de las actuaciones cofinanciadas por los Fondos estructurales y se analizan los resultados obtenidos. Finalmente, se concreta en las actuaciones dirigidas al desarrollo de la sociedad del conocimiento y se exponen las conclusiones.

\section{POLÍTICA DE COHESIÓN ECONÓMICA Y SOCIAL EN LA UE: UNAS POCAS CUESTIONES BÁSICAS}

La política de cohesión económica y social de la UE es, junto con el mercado único y la unión monetaria, uno de los tres pilares en los que se basa la construcción de un espacio económico y político europeo (Comisión Europea, 2007). La reforma de los Fondos estructurales de 1988, y el consiguiente refuerzo de esta política, transformó la naturaleza del modelo de integración europeo, 
pasando de un modelo que ponía el énfasis, fundamentalmente, en la integración económica de los mercados a otro que complementa esta integración con la incorporación de un principio de solidaridad territorial. Se buscan simultáneamente integración y cohesión. Con la búsqueda de una mayor cohesión entre Estados miembros y regiones se persigue, en última instancia, que los beneficios derivados de la integración económica lleguen a todos los ciudadanos y grupos sociales de la UE.

El hecho de que el modelo de integración económica europeo constituya también un modelo de solidaridad territorial suponía un indudable atractivo añadido para aquellos países europeos menos desarrollados que se han ido integrando hasta ahora en la UE, así como para los que desean adherirse en un futuro más o menos próximo. Participar -y beneficiarse dada su situación socioeconómica- en la política de cohesión es visto como un medio para alcanzar un crecimiento económico sostenible a medio y largo plazo. Con los recursos destinados a esta política se financian inversiones en capital físico, humano y tecnológico imprescindibles para elevar la capacidad competitiva. Éste es precisamente el objetivo estratégico básico de esta política comunitaria: conseguir que los países y regiones menos desarrollados sean competitivos y logren una mayor convergencia real. Tal y como señala la Comisión Europea (2004) en su tercer informe sobre la cohesión económica y social:

Es, pues, una política muy específica que implica una transferencia de recursos entre los Estados miembros a través del presupuesto de la Unión Europea con el fin de apoyar el crecimiento económico y el desarrollo sostenible por medio de la inversión en las personas y en el capital físico. Eso significa, asimismo, que el concepto de cohesión que se ha aplicado a escala europea no ha sido un concepto pasivo que redistribuya la renta, sino una política dinámica que trata de crear recursos seleccionando los factores de competitividad económica y de empleo, especialmente allí donde el potencial inutilizado es elevado (p. 25).

La importancia de esta política queda reflejada en su peso dentro del Presupuesto General de la UE, donde lleva más de una década absorbiendo aproximadamente un tercio del mismo. Esto la convierte en la actualidad en la principal política común desde el punto de vista del gasto comunitario, pasando por encima de la que siempre había sido la primera política dentro del Presupuesto: la política agraria común (PAC). A su vez, dentro de la política de cohesión, son las regiones menos desarrolladas las que reciben la mayor parte de los recursos (entre un 60 y un $70 \%$ del total). 


\begin{tabular}{l|l}
\hline Alemania & $\begin{array}{l}\text { Brandenburg, Mecklenburg-Vorpommern, Sachsen, Sachsen-Anhalt, Thüringen, Berlín } \\
\text { Este }\end{array}$ \\
\hline Austria & Burgenland \\
\hline Belgica & Hainut \\
\hline España & $\begin{array}{l}\text { Galicia, Principado de Asturias, Castilla y León, Castilla-La Mancha, Extremadura, } \\
\text { Comunidad Valenciana, Andalucía, Murcia, Ceuta, Melilla, Canarias, Cantabria }\end{array}$ \\
\hline Finlandia & Itä-Suomi, Väli-Suomi (en parte), Pohjois-Suomi (en parte) \\
\hline Francia & Guadeloupe, Martinique, Guyane, Reunión, Córcega, Nord-Pas-de-Calais \\
\hline Grecia & todo el territorio nacional \\
\hline Irlanda & Border, Midland and Western, Southern and Eastern \\
\hline Italia & Campania, Puglia, Basilicata, Calabria, Sicilia, Cerdeña, Molise \\
\hline Países Bajos & Flevoland \\
\hline Portugal & Norte, Centro, Alentejo, Algarve, Azores, Madeira, Lisboa, Vale do Tejo \\
\hline Reino Unido & $\begin{array}{l}\text { South Yorkshire, West Wales and the Valleys, Cornwall and Isles of Scilly, Merseyside, } \\
\text { Northern Ireland, Highlands and Islands }\end{array}$ \\
\hline Suecia & Norra Mellansverige (en parte), Mellersta Norrland (en parte), Övre Norrland (en parte) \\
\hline Cuadro - & Objut no \\
\hline
\end{tabular}

Cuadro 1 - Regiones Objetivo ${ }^{\circ} 1$ y para la UE-15 ${ }^{(*)}(2000-2006)$

Fuente: Preparado por los autores

En este tipo de regiones se incluyen, fundamentalmente, aquellas que tienen un PIB per capita inferior al 75\% de la media europea, si bien en el periodo objeto de estudio en este artículo (20002006) se incluían también las regiones ultraperiféricas y las regiones nórdicas con muy baja densidad de población. En la actualidad, reciben el nombre de regiones Objetivo Convergencia, mientras que en el periodo de programación 2000-2006, se denominaban regiones Objetivo n ${ }^{\circ} 1$. En el Cuadro 1 se recogen estas últimas, con la excepción de las de los países que se incorporaron a la UE en la ampliación de 2004, pertenecientes mayoritariamente a la Europa del Este.

Para acceder a los recursos financieros que la UE destina a esta política, las acciones a financiar deben ser objeto de una programación plurianual. En la elaboración de los distintos planes y programas de desarrollo participan activamente no sólo las autoridades comunitarias, sino también las de los 
Estados miembros afectados, tanto a nivel nacional como regional. De hecho, son los propios Estados miembros los que realizan un análisis DAFO de su situación socioeconómica y plantean cual debería ser la estrategia a seguir, así como las necesidades financieras de la misma. La Comisión Europea analiza, negocia en caso necesario con los Estados miembros posibles modificaciones y da, finalmente, luz verde a estos documentos. En concreto, los que recogen las medidas específicas a ejecutar y la financiación de las mismas son los Programas Operativos (PO).

\section{BASE DE DATOS UTILIZADA}

El análisis de las actuaciones dirigidas a potenciar la sociedad del conocimiento y desarrolladas en el marco de la política de cohesión económica y social de la UE, que se presenta en este trabajo, se ha llevado a cabo en el ámbito de las regiones europeas Objetivo nº 1 y se refiere al periodo 2000-2006.

El estudio ha sido realizado, por lo tanto, a partir de los Programas Operativos correspondientes a las regiones Objetivo $\mathrm{n}^{\circ} 1$ de los Estados miembros de la UE-15 (antes de las dos últimas ampliaciones) con la excepción de todas las regiones griegas, suecas y finlandesas y de dos de los Departamentos franceses de ultramar: Martinica y Guadalupe. Mientras que en el caso de estos últimos no se pudieron localizar los documentos completos correspondientes a sus PO, la exclusión de las regiones de Grecia, Suecia y Finlandia tuvo que ver con las dificultades lingüísticas a la hora de recabar la información necesaria para el desarrollo de este trabajo.

Si bien es posible encontrar resúmenes en inglés de todos los PO, los documentos completos se encuentran únicamente en el idioma del país de que se trata y esto, en el caso de los idiomas griego, sueco y finlandés, resultó ser una barrera insalvable. Es, precisamente, en estos documentos donde se encuentra la información necesaria para nuestro análisis, tanto cualitativa (referente a la descripción y detalle de las medidas concretas a ejecutar) como cuantitativa (datos de coste total de los distintos ejes y medidas, de gasto público y de financiación comunitaria). No obstante, contar con los datos de los $\mathrm{PO}$ de las restantes regiones Objetivo ${ }^{\circ} 1$ nos ha permitido recopilar un volumen de información muy significativo y lo suficientemente representativo del conjunto de regiones europeas menos desarrolladas como para hacer posible el análisis que se presenta en este trabajo.

La estrategia de desarrollo de los PO se estructura en ejes prioritarios y medidas. Cada uno de los ejes prioritarios se refiere a un determinado ámbito de actuación, de modo que incluye una serie de medidas o acciones concretas relacionadas con el mismo. Hemos encontrado que la clasificación y definición de ejes y medidas de los PO, especialmente en el nivel más desagregado correspondiente a 
las medidas, no son iguales en todos los países analizados. Por ello, una primera tarea ha sido la homogeneización de todos los PO con los que se ha trabajado. Esto se hizo definiendo un conjunto de medidas dentro de las cuales se incluyeron las actuaciones especificadas en los PO analizados. Se elaboró así una base de datos propia. Todas las actuaciones cofinanciadas dentro de los PO analizados se incluyen en alguna de las medidas anteriores. Estas están, a su vez, agrupadas en siete categorías o ejes de actuación:

- Mejora de la competitividad y desarrollo del tejido productivo, donde se incluyen aquellas actuaciones dirigidas a apoyar a las empresas en la región, tanto por medio de ayuda económica como de la provisión de toda una serie de espacios, equipamientos y servicios necesarios para el desarrollo de su actividad productiva.

- Sociedad del conocimiento, eje en el que se recogen las medidas de fomento de la I+D y la innovación, así como las dirigidas a impulsar la sociedad de la información.

- Medioambiente, entorno natural y recursos hídricos, objeto de estudio de nuestro análisis.

- Formación, educación y empleo, que incluye desde la creación y mejora de infraestructuras educativas hasta políticas activas en el mercado de trabajo.

- Desarrollo local y urbano, conjunto de actuaciones a desarrollar tanto en áreas urbanas como en municipios de menor tamaño y dirigidos a mejorar la calidad de vida de sus habitantes, pero también a incrementar el atractivo de estos lugares para el desarrollo de la actividad económica.

- Redes de transporte y energía, que recoge las inversiones a realizar en infraestructuras de comunicaciones y energéticas.

- Agricultura y desarrollo rural, donde se encuentran medidas de apoyo, fomento y mejora de las condiciones en que se desarrolla la actividad agrícola y de desarrollo de las zonas rurales, especialmente a través de la diversificación de las actividades productivas en las mismas.

Como ya se ha indicado, el presente trabajo se centra en uno de los ejes de actuación definidos: Sociedad del conocimiento. Las medidas incluidas en el mismo se detallan a continuación:

- Estrategias de innovación regional/Sistemas Regionales de Innovación, incluyendo todos aquellos aspectos relacionados con el fomento de redes, cooperación, capital social, etc. que tienen una influencia positiva sobre el aprendizaje colectivo y la innovación en un entorno regional.

- Proyectos de investigación, innovación y desarrollo tecnológico, donde la UE contribuye a la cofinanciación de los mismos. 
- Equipamiento científico-tecnológico y formación en I+D. En esta medida se pueden diferenciar dos grupos de actuaciones que tienen un nexo común: generar capacidades.

- Centros públicos de investigación y centros tecnológicos, una medida claramente orientada a la creación de infraestructuras.

- Sociedad de la información: en esta medidas se recogen todas las actuaciones dirigidas al desarrollo de las Tecnologías de la Información y la Comunicación (TICs)

Estas medidas se pueden dividir en dos grupos:

a) Acciones destinadas a fomentar la creación y transferencia de tecnología (medidas 1 y 2).

b) Acciones dirigidas a la creación de infraestructuras (medidas 3, 4 y 5).

\section{ANÁLISIS ESTADÍSTICO Y RESULTADOS}

El punto de partida del análisis estadístico realizado fue comprobar la existencia de una relación directa o inversa entre los siete ejes de actuación de los PO y el crecimiento del PIB per capita durante el periodo 2000-2006 en las 43 regiones para las que, finalmente, se realizó el estudio, puesto que se tuvieron que excluir Reunión y Berlín Este. En el primer caso, porque al realizar el análisis estadístico la región presentaba un comportamiento atípico (outlier). En el segundo caso, no se ha incluido Berlín Este debido a que los datos del PIB per capita están disponibles sólo para Berlín en su conjunto.

Se calculó el coeficiente de correlación lineal, que dio como resultado la existencia de una correlación positiva entre la tasa de variación del PIB per capita en el periodo considerado y el gasto total de los PO, por un lado, y de los ejes 3, 5, 6 y 7, por otro. El resultado es similar si en lugar de trabajar con la tasa de variación del PIB per capita se utilizan logaritmos (ver Cuadro 2).

El análisis de correlaciones realizado muestra que los recursos comunitarios destinados a financiar actuaciones medioambientales (eje 3), desarrollo local y urbano (eje 5), infraestructuras de transporte y energía (eje 6) y agricultura y desarrollo rural (eje 7) han tenido una incidencia positiva en la evolución de los niveles de renta per capita de las regiones beneficiadas. No es así en el caso de las medidas dirigidas a mejorar la competitividad y el desarrollo del tejido productivo (eje 1), a la sociedad del conocimiento (eje 2) y a formación, educación y empleo (eje 4). 


\begin{tabular}{l|c|c}
\hline VARIABLES & TASA 00-06 & Ln (PIB 06 / PIB 00 $)$ \\
\hline COSTE & 0.1527 & 0.0450 \\
\hline Eje 1 & -0.5682 & -0.4731 \\
\hline Eje 2 & -0.3740 & -0.3398 \\
\hline Eje 3 & 0.3141 & 0.3298 \\
\hline Eje 4 & -0.4151 & -0.4758 \\
\hline Eje 5 & 0.1578 & 0.1663 \\
\hline Eje 6 & 0.6265 & 0.5705 \\
\hline Eje 7 & 0.1562 & 0.1081 \\
\hline
\end{tabular}

Cuadro 2 - Análisis de correlaciones

Fuente: Preparado por los autores

Este resultado, en principio sorprendente, requiere la búsqueda de explicaciones al mismo, pues parece que determinadas actuaciones de política regional no producen los efectos deseados. En relación con las actuaciones dirigidas a mejorar la competitividad y el desarrollo del tejido productivo (eje 1), hay que tener en cuenta que el grueso de las mismas consiste en la concesión de ayudas y subvenciones a empresas. Este tipo de incentivo económico es uno de los instrumentos utilizados tradicionalmente por la política regional y, aunque se mantiene, su eficacia ha sido y es cada vez más cuestionada.

Más difícil de explicar parece el resultado relativo al gasto en $\mathrm{I}+\mathrm{D}+\mathrm{i}$ y a la sociedad de la información (eje 2) y a la formación de los recursos humanos (eje 4). En lo que se refiere al eje 2, hay que tener en cuenta que se trata de un capítulo de gasto de indudable importancia para la competitividad regional, pero que produce efectos a medio y largo plazo. No obstante, el Cuarto Informe realiza una crítica en torno a la forma en que se ha planteado la política de I+D+i en el marco de la cohesión. En concreto, se refiere al énfasis puesto en la construcción de infraestructuras en este ámbito, dando menos importancia a la creación y desarrollo de las conexiones entre empresas y universidades y centros de investigación. Se indica, además, que ésta puede ser en parte la explicación de por qué los esfuerzos realizados en transferencia de tecnología no han producido todavía los resultados que cabría esperar (Comisión Europea, 2007).

En el caso de la formación de los recursos humanos, para obtener el efecto deseado tiene que haber una conexión entre las necesidades reales de las actividades productivas regionales y la oferta de formación realizada. Si esa conexión no existe, el esfuerzo invertido no se traduce en mayor empleo y crecimiento económico. 
El siguiente paso ha sido contrastar estadísticamente la existencia de un efecto positivo de cada uno de los ejes sobre la tasa de crecimiento del PIB per capita de las regiones consideradas. Para ello se plantea un modelo econométrico en el que se incluyen como variables explicativas el gasto total de los PO y el peso de cada uno de los ejes de actuación en los que éstos se dividen.

$$
g_{j}=\beta_{1}+\beta_{2} \operatorname{COSTE}+\beta_{3} \text { GASTOID }_{04}+\sum_{j=4}^{11} \beta_{j} \mathrm{EJE}_{j}{ }^{+} \varepsilon_{j}
$$

La estimación de un modelo completo que incorpore todas las variables explicativas no arroja resultados estadísticamente significativos, por lo que se prescinde de las variables no relevantes, obteniendo dos modelos, M1 y M2, con un comportamiento aceptable que se resumen en el Cuadro 3.

\begin{tabular}{c|c|c}
\hline VARIABLES & M1 & M2 \\
\hline Coste & $0.00003^{* *}$ & $0.00002^{*}$ \\
& $(0.0001)$ & $(0.0001)$ \\
\hline Eje 2 & 0.0061 & \\
\hline \multirow{2}{*}{ Eje 5 } & $(0.0042)$ & $0.0035^{*}$ \\
\hline Eje 6 & $0.0048^{*}$ & $(0.0022)$ \\
\hline Eje 7 & $(0.0023)$ & $0.0066^{* * *}$ \\
\hline \multirow{2}{*}{ Constante } & $0.0078^{* * *}$ & $(0.0010)$ \\
\hline $\mathbf{R}^{2}$ & $(0.0013)$ & $0.0038^{*}$ \\
\hline $\mathbf{R}^{2}$ ajustado & $0.0044^{*}$ & $(0.0023)$ \\
\hline Root MSE & $(0.0023)$ & $\left(0.1019^{* *}\right.$ \\
\hline
\end{tabular}

Cuadro 3 - Estimaciones con los ejes de actuación y el coste de los programas

Fuente: Preparado por los autores

En el Modelo 1, se observa una influencia positiva de los ejes 2, 5, 6 y 7 sobre la tasa de variación del PIB per capita. Estos resultados son coherentes con lo esperado a partir del análisis de correlaciones realizado inicialmente, salvo en el caso del eje 3 que ahora no es relevante a pesar de su correlación inicial positiva, y del eje 2, que ahora sí lo es. Este último eje presenta un nivel crítico que no es muy bajo, pero siendo no excesivamente estrictos desde el punto de vista estadístico, podría ser 
incluido en el análisis económico (Modelo 1). Y es que, a pesar de la correlación negativa encontrada previamente entre el eje 2 y la tasa de variación del PIB per capita, en relación con la cual ya se han apuntado algunas explicaciones, es indudable que el gasto realizado en infraestructuras de I+D+i tiene que tener un efecto evidente en el crecimiento del PIB regional. En la Tabla 2 se presentan también resultados de la estimación realizada excluyendo el eje 2 (Modelo 2).

El resultado obtenido con respecto al eje 3 (medio ambiente, entorno natural y recursos hídricos) no es incoherente desde el punto de vista económico. Las actuaciones de los PO en este ámbito van dirigidas principalmente a protección y regeneración de suelos, espacios naturales, paisajes y al desarrollo y mejora de infraestructuras, como el abastecimiento de aguas o la gestión de residuos tanto urbanos como industriales. En definitiva, actuaciones que tienen un claro impacto positivo sobre la calidad de vida de la población, pero que tienen un impacto menos claro sobre el crecimiento de la renta per capita, especialmente a corto plazo.

\section{ANÁLISIS DE LAS ACCIONES DESTINADAS A LA SOCIEDAD DEL CONOCIMIENTO Y CONCLUSIONES}

El crecimiento económico y la convergencia de las regiones menos desarrolladas, para que sean sostenibles a largo plazo, deben estar basados en la mejora de la competitividad. Si se analizan los factores determinantes de ésta, se aprecian progresos sustanciales en infraestructuras básicas, como el transporte o el medio ambiente, así como en otras áreas donde los desequilibrios territoriales son particularmente pronunciados, como la I+D, la innovación, el acceso a la sociedad de la información o el capital humano (Argüelles y Benavides, 2008).

La política de cohesión con respecto a la I+D+i y a la sociedad de la información se orienta, en el periodo analizado, hacia la creación de capacidades e infraestructuras en este ámbito, especialmente en las regiones del Objetivo $\mathrm{n}^{\circ} 1$. La proporción de Fondos estructurales asignados a I+D+i varió, sin embargo, mucho entre las regiones, desde menos de un $5 \%$ en la mayor parte de la Europa meridional y las regiones ultraperiféricas, hasta más del 15\% en las regiones nórdicas (Comisión Europea, 2007, p. 106).

En el Cuadro 4 se presentan los datos correspondientes a la participación del Eje 2 en el coste total de los PO de los Fondos estructurales para el periodo 2000-2006 en las regiones analizadas. Se puede apreciar cómo los porcentajes más bajos corresponden a las regiones de España, Portugal, Italia y Francia, mientras que los más elevados corresponden a regiones de Alemania, Austria, Bélgica, la 
región sureste de Irlanda y algunas británicas. Aún perteneciendo todas ellas al grupo de regiones menos desarrolladas a nivel europeo, se aprecia un efecto país: hay una menor incidencia de este gasto en el coste total de los PO en las que pertenecen a los países más meridionales.

\begin{tabular}{|c|c|c|c|c|c|c|c|}
\hline & \multirow{2}{*}{$\begin{array}{l}\text { Coste } \\
\text { Total del } \\
\text { PO (mill. } \\
\text { euros) }\end{array}$} & \multirow{2}{*}{$\begin{array}{l}\% \text { del } \\
\text { Eje } 2 \\
\text { en el } \\
\text { Coste } \\
\text { Total } \\
\end{array}$} & \multicolumn{5}{|c|}{ Medidas del Eje 2: sociedad del conocimiento } \\
\hline & & & Medida 1 & Medida 2 & Medida 3 & Medida 4 & Medida 5 \\
\hline AUS Burgenland & 883.63 & 10.76 & 5.10 & 12.19 & 0.00 & 66.34 & 16.36 \\
\hline $\begin{array}{l}\text { AL Berlin Este } \\
\text { Branderburg } \\
\text { Mecklenburg- V. } \\
\text { Sachsen-Anhalt } \\
\text { Sachsen } \\
\text { Thüringen } \\
\end{array}$ & $\begin{array}{l}1078.79 \\
3090.00 \\
3674.20 \\
8550.70 \\
4785.21 \\
7014.58 \\
\end{array}$ & $\begin{array}{r}21.57 \\
7.23 \\
3.07 \\
10.74 \\
10.83 \\
12.72 \\
\end{array}$ & $\begin{array}{c}0.00 \\
2.98 \\
62.65 \\
0.00 \\
4.87 \\
0.00 \\
\end{array}$ & $\begin{array}{c}54.33 \\
24.10 \\
0.00 \\
40.96 \\
71.81 \\
42.40 \\
\end{array}$ & $\begin{array}{c}0.00 \\
28.18 \\
0.00 \\
27.17 \\
3.53 \\
16.59 \\
\end{array}$ & $\begin{array}{l}45.67 \\
28.18 \\
32.51 \\
27.17 \\
17.55 \\
7.48 \\
\end{array}$ & $\begin{array}{c}0.00 \\
16.55 \\
4.84 \\
4.70 \\
2.42 \\
33.53 \\
\end{array}$ \\
\hline BEL Hainut & 2264.21 & 12.70 & 5.91 & 44.60 & 13.44 & 36.05 & 0.00 \\
\hline $\begin{array}{l}\text { ESP Andalucía } \\
\text { Asturias } \\
\text { Canarias } \\
\text { Castilla Mancha } \\
\text { Castilla León } \\
\text { C. Valenciana } \\
\text { Extremadura } \\
\text { Galicia } \\
\text { Murcia } \\
\text { Cantabria } \\
\end{array}$ & $\begin{array}{r}10701.56 \\
2049.69 \\
2806.51 \\
3020.40 \\
4541.27 \\
4162.44 \\
3015.04 \\
5075.68 \\
1809.07 \\
518.05 \\
\end{array}$ & $\begin{array}{l}3.04 \\
2.20 \\
4.26 \\
7.43 \\
1.62 \\
8.57 \\
4.56 \\
3.38 \\
2.64 \\
7.43 \\
\end{array}$ & $\begin{array}{c}5.10 \\
0.00 \\
32.10 \\
27.48 \\
44.44 \\
9.97 \\
28.51 \\
18.26 \\
13.30 \\
0.00 \\
\end{array}$ & $\begin{array}{c}12.19 \\
15.46 \\
11.04 \\
13.72 \\
3.49 \\
33.46 \\
18.03 \\
25.06 \\
21.67 \\
0.00 \\
\end{array}$ & $\begin{array}{c}0.00 \\
13.40 \\
16.98 \\
0.00 \\
0.00 \\
15.40 \\
3.44 \\
2.01 \\
6.23 \\
33.33 \\
\end{array}$ & $\begin{array}{c}66.34 \\
15.46 \\
3.39 \\
7.62 \\
0.00 \\
15.32 \\
4.81 \\
7.02 \\
0.00 \\
0.00 \\
\end{array}$ & $\begin{array}{l}16.36 \\
55.69 \\
36.50 \\
51.18 \\
52.08 \\
25.85 \\
45.21 \\
47.65 \\
58.80 \\
66.67 \\
\end{array}$ \\
\hline $\begin{array}{l}\text { FR Reunion } \\
\text { Martinique } \\
\text { Guayane } \\
\text { Guadeloupe } \\
\text { Corse } \\
\text { Nord-Pas-Calais }\end{array}$ & $\begin{array}{r}3033.52 \\
\text { n.d. } \\
720.49 \\
\text { n.d. } \\
392.33 \\
1090.54 \\
\end{array}$ & $\begin{array}{r}1.47 \\
\text { n.d. } \\
6.30 \\
\text { n.d. } \\
5.03 \\
8.30 \\
\end{array}$ & $\begin{array}{c}0.00 \\
\text { n.d } \\
0.00 \\
\text { n.d. } \\
0.00 \\
53.59 \\
\end{array}$ & $\begin{array}{c}0.00 \\
\text { n.d. } \\
96.65 \\
\text { n.d. } \\
39.94 \\
11.46 \\
\end{array}$ & $\begin{array}{l}0.00 \\
\text { n.d. } \\
0.00 \\
\text { n.d. } \\
0.00 \\
0.00 \\
\end{array}$ & $\begin{array}{l}0.00 \\
\text { n.d. } \\
0.00 \\
\text { n.d. } \\
0.00 \\
0.00 \\
\end{array}$ & $\begin{array}{l}100.00 \\
\text { n.d. } \\
3.35 \\
\text { n.d. } \\
60.06 \\
34.96 \\
\end{array}$ \\
\hline $\begin{array}{l}\text { IRL Border Mid. \& W } \\
\text { Southern \& E R. }\end{array}$ & $\begin{array}{l}4091.49 \\
1729.91 \\
\end{array}$ & $\begin{array}{r}9.28 \\
19.10 \\
\end{array}$ & $\begin{array}{l}0.00 \\
8.37 \\
\end{array}$ & $\begin{array}{c}39.94 \\
0.00\end{array}$ & $\begin{array}{l}0.00 \\
0.00\end{array}$ & $\begin{array}{l}0.00 \\
0.00 \\
\end{array}$ & $\begin{array}{l}60.06 \\
91.63 \\
\end{array}$ \\
\hline $\begin{array}{l}\text { IT Basilicata } \\
\text { Calabria } \\
\text { Campania } \\
\text { Puglia } \\
\text { Sardegna } \\
\text { Sicilia } \\
\text { Molise } \\
\end{array}$ & $\begin{array}{r}1680.52 \\
5262.99 \\
7681.13 \\
6693.84 \\
4235.07 \\
8433.44 \\
462.77 \\
\end{array}$ & $\begin{array}{r}5.68 \\
2.49 \\
8.41 \\
17.90 \\
4.08 \\
6.18 \\
6.33 \\
\end{array}$ & $\begin{array}{c}23.33 \\
34.37 \\
35.16 \\
67.33 \\
0.00 \\
25.18 \\
0.00 \\
\end{array}$ & $\begin{array}{c}0.00 \\
0.00 \\
25.22 \\
5.70 \\
45.21 \\
0.00 \\
26.28 \\
\end{array}$ & $\begin{array}{l}0.00 \\
0.00 \\
0.00 \\
0.00 \\
0.00 \\
5.02 \\
0.00 \\
\end{array}$ & $\begin{array}{l}0.00 \\
0.00 \\
0.00 \\
0.00 \\
0.00 \\
0.00 \\
0.00\end{array}$ & $\begin{array}{l}76.67 \\
65.63 \\
39.62 \\
26.97 \\
54.79 \\
69.80 \\
73.72 \\
\end{array}$ \\
\hline HOL Flevoland & 464.26 & 8.21 & 71.52 & 0.00 & 0.00 & 0.00 & 28.48 \\
\hline PT Azores & 1295.50 & 1.40 & 7.31 & 9.90 & 9.90 & 9.90 & 62.97 \\
\hline
\end{tabular}

Revista de Administração e Inovação, São Paulo, v. 7, n. 3, p. 44-57, jul./set. 2010 


\begin{tabular}{l|r|r|c|c|c|c|cc}
\hline Algarve & 755.64 & 2.26 & 0.00 & 26.12 & 0.00 & 0.00 & 73.88 \\
Alentejo & 2032.68 & 2.48 & 45.45 & 0.00 & 0.00 & 6.51 & 48.04 \\
Centro & 2829.13 & 2.14 & 0.00 & 13.18 & 0.00 & 0.00 & 86.82 \\
Madeira & 1216.89 & 3.38 & 63.24 & 0.00 & 0.00 & 0.00 & 36.76 \\
Norte & 4854.40 & 1.51 & 33.35 & 9.74 & 0.00 & 0.00 & 56.91 \\
Lisboa e Vale Tejo & 2728.54 & 8.31 & 85.96 & 3.08 & 0.00 & 0.00 & 10.96 \\
\hline & & & & & & & \\
RU Cornwall \& I. Scilly & 1323.51 & 8.27 & 91.16 & 8.84 & 0.00 & 0.00 & 0.00 \\
Merseyside & 3366.02 & 6.37 & 100.00 & 0.00 & 0.00 & 0.00 & 0.00 \\
South Yorkshire & 3052.85 & 15.75 & 76.37 & 0.00 & 0.00 & 0.00 & 23.63 \\
West Wales & 3988.12 & 13.61 & 14.27 & 21.55 & 21.55 & 0.00 & 42.62 \\
Highlands \& Islan. & 843.76 & 6.33 & 32.12 & 0.00 & 0.00 & 0.00 & 67.88 \\
Northern Ireland & 1486.26 & 9.15 & 50.98 & 12.74 & 0.00 & 0.00 & 36.27 \\
\hline
\end{tabular}

Cuadro 4 - Participación de las medidas de las acciones del Eje 2 en el coste total de las acciones de dicho Eje, en $\%$

Fuente: Preparado por los autores

En cuanto a las medidas, éstas habían sido divididas en dos grupos: acciones destinadas a fomentar la creación y transferencia de tecnología (medidas 1 y 2) y acciones dirigidas a la creación de infraestructuras (medidas 3, 4 y 5).

Una crítica realizada en torno a la forma en que se ha planteado el apoyo a la I+D+i en el contexto de la política regional europea, se refiere a que se ha puesto el énfasis en la construcción de infraestructuras, dando menos importancia a la aplicación de aquellas medidas necesarias para mejorar las relaciones entre las empresas y las universidades y los centros de investigación. Se indica, además, que ésta puede ser en parte la explicación de por qué los esfuerzos realizados en transferencia de tecnología no han producido todavía los resultados que cabría esperar (Comisión Europea, 2007, p. 107). Este hecho se puede apreciar de nuevo en muchas de las regiones Objetivo $\mathrm{n}^{\circ} 1$ estudiadas para el periodo 2000-2006. Volviendo al Cuadro 4, donde se recoge el peso de las distintas medidas del eje 2, se observa cómo en muchas de estas regiones el gasto se concentra en la segunda categoría de acciones, es decir, aquellas dirigidas a crear infraestructuras, sobre todo TICs. Esto es especialmente intenso en España y Portugal.

Ahora bien, es necesario situarse en el contexto de estas regiones, donde el énfasis puesto en la creación de infraestructuras es una estrategia lógica cuando existe un déficit claro de las mismas. Desarrollar la capacidad necesaria para innovar, transferir y asimilar conocimientos, es aquí un segundo paso que por supuesto también es necesario dar. La nueva política de cohesión económica y social de la UE para el periodo 2007-2013 basa su desarrollo en las Directrices Estratégicas Comunitarias en materia de cohesión que hacen referencia a convertir Europa en un lugar más 
atractivo para invertir y trabajar, mejorar los conocimientos y la innovación y crear más y mejores puestos de trabajo. En todo ello está implícita la inmersión en la economía del conocimiento.

\section{REFERENCIAS}

Argüelles, M. \& Benavides, C. (2008). Economía y política regional. Madrid: Delta.

Comisión Europea. (2007). Crecimiento de las regiones, desarrollo de Europa: cuarto informe sobre la cohesión económica y social. Luxemburgo: Oficina de Publicaciones Oficiales de las Comunidades Europeas.

Comisión Europea. (2004). Una nueva asociación para la cohesión convergencia competitividad cooperación: tercer informe sobre la cohesión económica y social. Luxemburgo: Oficina de Publicaciones Oficiales de las Comunidades Europeas.

European Commission. (n.d.). Regional policy-inforegio. Recuperado em 12 agosto, 2010, de http://ec.europa.eu/regional_policy/country/prordn/index_en.cfm?gv_pay=ES\&gv_reg=ALL\&gv_ obj=ALL\&gv_the=ALL\&gv_per=1.

Eurostat. News Release. Recuperado em 23 outubro, 2009, de http://epp.eurostat.ec.europa.eu/portal/page/portal/eurostat/home/.

Union Européenne. (2003). Les transports aériens dans les pays candidats en 2001 (Statistiques en bref, 1). Luxemburgo: Office des publications officielles des Communautés européennes. 


\title{
INNOVATION AND TECHNOLOGICAL DEVELOPMENT IN THE REGIONAL POLICY OF THE EUROPEAN UNION, 2000-2006. AN ANALYSIS OF THE LESS DEVELOPED
}

\begin{abstract}
The knowledge triangle - research-education-innovation is the basis of European efforts cope with Lisbon strategy objectives: to make the European Union the most competitive and dynamic economy, based on knowledge, in the world. To reach its objectives, EU use different policies, among them the economic and social cohesion policy. The target of this policy is to reduce disparities among European regions, by promoting the development of less developed regions and of those experiencing serious problems of structural transformation. These regions are the scope of this study, with special attention to Spain and Portugal. In these countries we analyze the operating program of structural funds during the last reported period: 2000-2006. This paper objective thus, is to evaluate the impact of efforts developed for creation and improvement of regional capabilities towards research and innovation, including the new TICs, over the regional economic growth. However, the development of a knowledge society is the only end of investments for the regional development studied, but we also looked at the different axes of the investment, such as improvement in competitiveness, in especial SMEs, the protection of environment, transportation and energy infra-structure and local and urban or rural development.
\end{abstract}

Keywords: Technological development; Innovation; Innovation policy; Regional development.

Data do recebimento do artigo: 02/07/2010

Data do aceite de publicação: 09/09/2010 